\title{
Competition, Product Proliferation and Welfare: A Study of the U.S. Smartphone Market*
}

\author{
Ying Fan $^{\dagger}$ \\ Chenyu Yang $\ddagger$ \\ University of Michigan \\ University of Michigan
}

February 12, 2016

\begin{abstract}
We develop and estimate a structural model of the U.S. smartphone market. Based on the estimates, we study (1) whether there are too few or too many products in the market from a welfare point of view; and (2) how competition affects product offerings in the market. We find that there are too few products and a reduction in competition further decreases the product offerings. These results suggest that merger policies should be stricter when we take into account the effect of merger on firms' product choices in addition to its effect on prices.
\end{abstract}

Key words: endogenous product choice, product variety, smartphone industry

JEL Classifications: L11, L15, L13, L63

\section{Introduction}

Product proliferation is common in many markets. In markets with vertical differentiation, firms often offer multiple products across the entire spectrum of quality. Firms do so because by offering a menu of products with different qualities, firms can induce consumers with different tastes for quality to sort themselves and hence extract more surplus. In an oligopoly market, product proliferation is also influenced by strategic considerations and is determined by firms' competition in the product space. Examples of such oligopoly markets include the printer market, the CPU market and the PC market. Another example is the smartphone market. For instance, in January 2013, there are 19 Samsung smartphones in the U.S. market, including Galaxy S III and Galaxy Note II at the high end of the quality spectrum, and Galaxy Appeal and Dart at the low end. In this paper, we study two questions about product proliferation in the U.S. smartphone market.

\footnotetext{
${ }^{*}$ We thank the Michigan Institute for Teaching and Research in Economics and NET Institute for their generous financial support.

${ }^{\dagger}$ Department of Economics, University of Michigan, 611 Tappan Street, Ann Arbor, MI 48109; yingfan@umich.edu

${ }^{\ddagger}$ Department of Economics, University of Michigan, 611 Tappan Street, Ann Arbor, MI 48109; chny.yang@gmail.com.
} 
This paper seeks to address two questions related to product proliferation. First, are there too few or too many products from a welfare point of view? Oligopolistic competition may lead to excessive product proliferation: a profit-maximizing firm will have a product in the market if the additional variable profit from doing so is larger than the corresponding additional fixed cost, but some of the variable profit increase comes from business stealing. Since firms do not take this negative externality (on other firms) into account, there can be too many products from a welfare point of view. On the other hand, different from a social planner, firms do not internalize consumer surplus when choosing their product offerings. If consumer surplus is increasing in the number of products, there may be too few products in the market. Therefore, it is an empirical question whether there are too few or too many products.

Secondly, we seek to understand how competition affects product offerings. When two firms merge, the merged firm internalizes the business stealing effect and thus may put fewer products in the market. At the same time, however, a merger also affects the pricing competition. Softened price competition may result in higher prices, which may increase the variable profit from adding a product, leading to more products in the market. All these changes eventually affect consumer surplus and producer surplus. In the second research question, we quantify the effect of competition on product variety, prices and welfare.

Combining these two research questions, this paper sheds light on how to adjust the leniency of competition policies when product offerings are endogenous. If competition leads to too many products, and merger reduces product offerings, then the merger policy should be more lenient because the consumer welfare loss due to potentially higher prices may be offset by the savings of fixed costs in the producer surplus. On the other hand, the policy should be stricter if merger reduces product offerings when there are already too few products in the market. We study these two questions in the context of the U.S. smartphone market. The smartphone industry has been one of the fastest growing and most innovative industries in the world with billions of dollars at stake. Samsung shipped out over 80 million smartphones in the third quarter of 2013 worldwide, and Apple more than 30 million. The industry has affected the daily lives of millions of consumers. Product proliferation is prominent in the smartphone industry. For example, in a given month between 2009 and March 2013, a smartphone handset maker on average offers 6 smartphones in the U.S. market. The average price dispersion among products offered by the same firm in a given month (measured in the standard deviation of the subsidized prices across products belong to the same firm/month, ${ }^{1}$ averaged across firms and months) is $\$ 47$ while the average price is $\$ 116$. The average price difference between a firm's highest-priced product and the lowest priced product, averaged across all firms and months, is $\$ 123$. Such within-firm price dispersion is associated with within-firm dispersion on product quality. Firms offer a wide selection of smartphones across the

\footnotetext{
${ }^{1}$ The subsidized price is the average retail price of a smartphone device that a carrier charges a consumer who uses its network service.
} 
quality spectrum. Firms provide this variety typically by simultaneously offering a few flagship products and a set of non-flagship products of lower qualities.

In this paper, we focus on how smartphone firms choose their non-flagship products in the U.S. market. We choose to study this particular aspect of product variety for several reasons. First, the flagship products are usually equipped with the cutting-edge technologies, which capitalize on the innovation processes of the many upstream industries that are sometimes beyond the control of the smartphone firms. Secondly, the large investment on developing a flagship product is, however, driven by the global market conditions. However, our data are only on the U.S. market, which accounts for about $10 \%$ of smartphone units sold in the global market. ${ }^{2}$ In contrast, non-flagship products are tailored to the U.S. market rather than sold globally. Having data on the U.S. market is enough to describe the demand of non-flagship products, and hence to study firms' product-choice decisions about them.

In order to study our research questions and quantify the welfare, we develop a structural model describing consumer demand and firms' product and price decisions. Specifically, the demand side is described by a random coefficient discrete choice model. In the model, the quality of a product is a linear function of a set of key product characteristics and consumers have heterogeneous tastes for quality. The supply side is captured by a static three-stage structural model. In the first stage, smartphone firms observe the exogenous qualities of the flagship products and make a decision about their non-flagship products. There is a fixed cost of having each product in the market. In the second stage, after observing the products offered by each firm and the realized demand and marginal cost shocks for each product, firms set the wholesale prices of all products - flagship or non-flagship - for carriers. Finally, carriers set the retail prices for consumers in the third stage.

Our data come from the Investment Technology Group (ITG) Market Research. This data set provides information on the price and quantity for all smartphones in the U.S. market between 2009 and March, 2013. For every carrier in the U.S. and every month during our sample period, we observe the price and sales of each smartphone sold through the carrier. We also observe key specifications of each product, such as battery talk time and camera resolution.

Using these data, we estimate consumers' demand for smartphones and firms' marginal cost following an estimation procedure similar to that in Berry, Levinsohn and Pakes (1995). We find that, on average, consumers value the battery talk time, the camera resolution, the generation of the chipset and the screen size. They also prefer a light-weight smartphone, ceteris paribus. The estimated coefficients are all statistically significant except for weight. We refer to the linear combination of product characteristics (the weights are given by the estimated coefficients of these characteristics) as the quality index of a product. We allow for a random coefficient on this quality index and find that the estimated standard deviation of this random coefficient is about $1 / 3$ of its mean, indicating a sizable consumer heterogeneity in their willingness-to-pay for quality. On the

\footnotetext{
${ }^{2}$ http://www.gartner.com/newsroom/id/2335616
} 
supply side, not surprisingly, we find that marginal cost is increasing in quality and decreasing over time.

We also obtain bounds on the fixed cost. The estimated demand and marginal cost functions, combined with an equilibrium model of a two-stage oligopoly pricing, determine a smartphone firm's variable profit for a given set of products. We obtain bounds on the fixed costs using the optimality condition of product offerings. Specifically, for any product in the market in a month, we obtain an upper bound of its fixed cost in that month, which is the difference between the firm's variable profit given the observed set of products and the variable profit if this product is removed. Intuitively, the fixed cost of a product must be bounded from above if the firm decides to offer it. Similarly, for any product that is not in the data, we can obtain a lower bound of its fixed cost. These bounds and the estimated demand and marginal cost are used for counterfactual simulations.

Counterfactual simulations are designed to address the two research questions of the paper. To answer the question of whether there are too few or too many products in the market, we conduct two counterfactual simulations for January, February and March 2013, the last three months of our data. We remove products in one set of counterfactual simulations and add products in the other. We find that even considering the maximum saving in the fixed cost from dropping a product (i.e., assuming that the fixed cost is at its upper bound), in all three months, total surplus decreases when we drop the lowest-quality product in each month. Consumers are worse off due to the decreased product offerings. This reduction in product offerings also hurt carriers. Smartphone firms as a whole, however, are better off, confirming the business-stealing effect of a product on competitors' profits. Nonetheless, the decrease in consumer surplus outweighs the internalization of business stealing effects, and removing a product is welfare reducing. Similarly, when we drop the lowest-quality product of each smartphone firm, the change in each welfare measure becomes larger but the overall effect has the same sign: total surplus decreases even when fixed costs are at their upper bounds. On the other hand, when we add the highest-quality discontinued product (i.e., a product that was in the previous month but is not in the given month), we find that consumer surplus increases by 3.7 million dollars in March 2013, for example. Carrier surplus increases by 2.8 million dollars, and smartphone firms' total variable profit increases by 1.6 million. For a product not in the data, we can only obtain a lower bound for its fixed cost, which is 1.5 million. Therefore, as long as the fixed cost is not too high, specifically, not more than 5 times of its lower bound, total surplus would increase with the increased product offerings. Overall, these counterfactual simulation results suggest that there are too few products, and that this is because the product variety effect dominates the business stealing effect.

Turning to the second research question of how competition affects product variety, we simulate the effect of a hypothetical merger between Samsung and LG in March 2013. Samsung and LG are the second and the third largest smartphone firms in terms of sales in March 2013. In this exercise, we compute the post-merger product choice equilibrium and pricing equilibrium. To keep 
the problem tractable, we restrict the set of potential products for each firm to be the firm's products in either February 2013 or March 2013. Since each firm decides on its product portfolio, its choice set consists of any subset (of any size) of its potential products. Thus, the choice set can be prohibitively large from a computational point of view. For example, if the number of potential products is 20 , the size of the choice set is more than 1 million. Therefore, we use a heuristic approach to find a firm's best-response product portfolio for given portfolios of the competitors. Starting from the pre-merger product portfolio, a firm finds its highest profit from dropping one product or adding one product. If no such deviation is profitable, we stop. Otherwise, starting from the most-profitable deviating product portfolio we just find, we again find whether the firm's profit can increase by adding or dropping one product. We continue this process until the firm's profit does not increase by such a one-product deviation. We embed this optimization routine in a best-response iteration to solve for the new equilibrium. Since we obtain only bounds of fixed costs, for each potential product, we randomly draw 5 fixed-cost values within a range consistent with the bounds of this product's fixed cost, and compute the post-merge equilibrium corresponding to each set of draws. We find that after the merger, the product offerings in the market decrease and the sales-weighted average retail price increases. Consequently, consumers are worse off and total welfare decreases. Combining with our finding regarding the first research question (i.e., there are too few products in the market), these results suggest that merger policies should be stricter when we take into account the effect of merger on product offerings.

By studying the welfare implication of product proliferation and how competition affects them, this paper is related to the literature of endogenous product choice. Examples in this literature include Mazzeo (2002), Draganska, Mazzeo and Seim (2009), Crawford, Shcherbakov and Shum (2015), Fan (2013), Eizenberg (2014) and Berry, Eizenberg and Waldfogel (forthcoming). ${ }^{3}$ Among these papers, Berry, Eizenberg and Waldfogel (forthcoming) is the most closely related to this paper as they also address the question of whether there is too much product variety. But different from their paper, which studies the radio industry where excessive product variety is equivalent to excessive firm entry, in our setting, product variety is determined not only by the set of firms in the market, but also by each firm's product choice given the market structure. Therefore, in addition to studying the question of whether there are too few or too many products, we also study how a change in market structure (specifically, competition) affects product variety, and eventually prices and welfare. This paper thus provides guidance on merger policies by addressing these two questions.

The rest of the paper is organized as follows. Section 2 describes our data. Section 3 presents the structural model of the smartphone market and derives estimating equations. Section 4 explains

\footnotetext{
${ }^{3}$ Other examples in this literature include Seim (2006), Watson (2009), Chu (2010), Crawford and Yurukoglu (2012), Sweeting (2013), and Nosko See Crawford (2012) for a survey of this literature. Examples in the theoretical literature on this topic include Johnson and Myatt (2003) and Shen, Yang and Ye (forthcoming).
} 
the estimation approach and reports the estimation results. Section 5 explains counterfactual simulations and shows the results. Finally, we conclude in Section 6 .

\section{Data}

Our data come from the Investment Technology Group (ITG) Market Research. This data set provides information on the price and quantity for all smartphones in the U.S. market between 2009 and March 2013. For every carrier in the U.S. and every month during our sample period, we observe the price and sales for each smartphone sold through the given carrier in the given month. We also observe key specifications of each product such as camera resolution and chipset generation.

The price information provided by the ITG for the four major national carriers (AT\&T, Verizon, Sprint and T-Mobile) is the average price for a smartphone device that a carrier charges a consumer who uses this carrier's network service. In other words, the price reported for the four major national carriers is the so-called subsidized price. ${ }^{4}$ The subsidized price for a smartphone is not the true cost of buying a smartphone from a consumer's perspective because the consumer also needs to pay for the service plan. As will be explained later, we therefore include a carrier/year-specific fixed effect to capture the average service cost for a consumer. The price information for carriers other than the four major national carriers (for example, Boost and MetroPCS) is unsubsidized, as these carriers very often only provide prepaid service plans. In addition, these carriers usually serve only one regional market. For these reasons, we drop observations of these fringe carriers. ${ }^{5}$

In the end, our sample consists of 3256 observations, each of which is a smartphone/carrier/month combination between 2009/01 and 2013/03. There are 18 firms and 277 smartphones in the sample. Among them, 47 smartphones are flagship smartphones. Flagship products are usually equipped with the cutting-edge technologies and are typically sold globally. For example, all iPhones are flagship smartphones. Majority of the smartphones, especially non-flagship smartphones, are sold through one carrier. Table 1 shows that $90 \%$ of smartphones and $93 \%$ of non-flagship smartphones are sold though one carrier. Moreover, when the same smartphone is sold through different carriers, it is often pre-installed with different softwares and sometimes sold at different prices. Therefore, we define a product as a smartphone/carrier combination.

Table 2 presents the summary statistics on quantity, price and product characteristics. From Table 2, we can see that the average monthly sales of a product is around 77,000 while the standard deviation of the monthly sales is about twice the mean, suggesting a large dispersion in the sales of

\footnotetext{
${ }^{4}$ Note that the carrier fee structure has been relatively stable during our sample period. In April 2013, however, TMobile launched an "Uncarrier" campaign, which abandoned service contracts and subsidies for devices. Consumers are asked to buy a device outright, or through a device installment plan. Other carriers followed suit after T-Mobile made this change.

${ }^{5}$ The total market share of these fringe carriers in terms of smartphones sold is about $10 \%$.
} 
Table 1: Carriers per Smartphone

\begin{tabular}{crrcrr}
\hline \multicolumn{3}{c}{ All Smartphones } & \multicolumn{3}{c}{ Non-flagship Smartphones } \\
\hline \# of Carriers & Count & Percent & \# of Carriers & Count & Percent \\
\hline \hline 1 & 249 & $89.89 \%$ & 1 & 214 & $93.04 \%$ \\
2 & 22 & $7.94 \%$ & 2 & 15 & $6.52 \%$ \\
3 & 4 & $1.44 \%$ & 3 & 1 & $0.43 \%$ \\
4 & 2 & $0.72 \%$ & & & \\
\hline
\end{tabular}

products. There is also a sizable variation in price across observations: the price is 122 dollars on average, with a standard deviation of 85 . For each product, we observe product characteristics such as battery talk time (a measure - admittedly not a perfect one - for battery efficiency), camera resolution, screen size measured by the diagonal of the screen, and weight. We also observe the generation of the chipset used by each product. For example, there are five Apple smartphones in our data (i.e., iPhone 3G, iPhone $3 \mathrm{Gs}$, iPhone 4 , iPhone $4 \mathrm{~s}$ and iPhone 5), each of which uses a chipset of a different generation. Table 2 reports summary statistics of these product characteristics. Their standard deviations are about $17 \%$ to $47 \%$ of the corresponding means, indicating that the market has a wide variety of products.

Table 2: Summary Statistics

\begin{tabular}{lllll}
\hline Variable & Mean & Std. Dev. & Min & Max \\
\hline \hline Quantity (1000) & 77.54 & 146.04 & 0.04 & 1419 \\
Price (\$) & 122.16 & 85.24 & 0 & 406.9 \\
Battery talk time (hours) & 7.08 & 2.93 & 3 & 22 \\
Camera resolution (megapixel) & 4.65 & 2.18 & $0^{a}$ & 13 \\
Chipset generation 2 & 0.23 & 0.42 & 0 & 1 \\
Chipset generation 3 & 0.25 & 0.43 & 0 & 1 \\
Chipset generation 4 & 0.14 & 0.34 & 0 & 1 \\
Chipset generation 5 & 0.09 & 0.29 & 0 & 1 \\
Screen size (inch) & 3.44 & 0.73 & 2.2 & 5.54 \\
Weight (gram) & 135.31 & 22.72 & 89.5 & 193 \\
\hline Obs & 3256 & & \\
\hline${ }^{a}$ One product in our sample (BlackBerry & $8830)$ does not have a camera.
\end{tabular}

Table 3 lists the top six firms according to their average monthly smartphone sales: Apple, Samsung, BlackBerry, HTC, Motorola and LG. Among them, Apple is the undisputed leader in the industry, with an average monthly sales of about 2 million. It is followed by Samsung, whose average sales in a month is 0.76 million. From Table 3 , we can see that all of these six firms offer multiple products simultaneously. For example, on average, Samsung has 13 products per month, followed by BlackBerry and HTC who offer 11 products per month.

The multiple products offered by a firm are of different qualities and are charged different prices, as shown by Table 4 on the within-firm/month dispersion of price and product characteristics. In 
Table 3: List of Top Five Smartphone Firms

\begin{tabular}{llccc}
\hline Firm & Headquarters & $\begin{array}{c}\text { Avg. Number } \\
\text { of Products }^{a}\end{array}$ & $\begin{array}{c}\text { Avg. Retail Price }^{b} \\
(\$)\end{array}$ & $\begin{array}{c}\text { Avg. Monthly Sales } \\
\text { (million) }\end{array}$ \\
\hline \hline Apple & U.S. & 3.94 & 166.19 & 1.99 \\
Samsung & Korea & 13.20 & 138.73 & 0.76 \\
BlackBerry & Canada & 11.31 & 162.29 & 0.61 \\
HTC & Taiwan & 11.90 & 168.35 & 0.60 \\
Motorola & U.S. & 7.90 & 180.62 & 0.46 \\
LG & Korea & 7.19 & 81.46 & 0.33 \\
\hline
\end{tabular}

${ }^{a}$ Averaged across months.

${ }^{b}$ Our data report the average price for each product/month. We compute the sales weighted average of this price across all product/months related to a firm, i.e., across all products of the firm and months that a product of this manufacturer is sold.

Table 4, we report two dispersion measures: standard deviation and range. Take price for example. For each firm/month, we compute the standard deviation of price across all products available in the given month produced by the given firm. We set the standard deviation to 0 for firm/months with a single product. We then take the average of these standard deviations across all 577 firm/months. Similarly, we compute the within-firm/month range as the difference between the highest and the lowest price among all products in the same firm/month and take the average across all 577 firm/months. We report these two average within-firm/month dispersion measures in Table 4 . For example, the average within-firm/month standard deviation in price is 42.42 dollars, which is about $1 / 3$ of the average price (122.16 dollars) in the data. In comparison, the standard deviation of price across all observations is 82 dollars (see Table 2), implying that the within firm/month variation in price is an important component of its total variation. The average range of the price within the same firm/month is as high as 123 dollars according to Table 4. The within firm variation of product characteristics is also significant. For example, looking at the range of chipset generation, we can see that smartphone firms on average simultaneously offer products whose chipsets are one generation apart. Overall, Table 4 provides evidence on product proliferation in this industry. In the next section, we set up a model to describe how firms choose their products and the prices of their products.

Table 4: Summary Statistics on Quality and Price Dispersion within a Firm/month

\begin{tabular}{lcc}
\hline & Average Std. Dev. & Average Range \\
\hline \hline Price (\$) & 42.42 & 122.5 \\
Battery talk time (hours) & 1.04 & 3.10 \\
Camera resolution (megapixel) & 0.81 & 2.16 \\
Chipset generation & 0.36 & 0.93 \\
Screen size (inch) & 0.21 & 0.61 \\
Weight (gram) & 11.12 & 32.23 \\
\hline
\end{tabular}




\section{Model}

\subsection{Demand}

The demand is described by a discrete choice model. In the model, a consumer chooses a smartphone product or an outside option of not buying a smartphone. The utility that consumer $i$ gets from purchasing product $j$ in period $t$ is assumed to be

$$
u_{i j t}=\beta_{i} q_{j}-\alpha p_{j t}+\kappa_{c(j) t}+\xi_{j t}+\varepsilon_{i j t}
$$

where $q_{j}$ is a quality index of product $j$. It depends on a set of product characteristics $\boldsymbol{x}_{j}$ such as camera resolution and a brand dummy. Specifically, we assume that quality is linear in the product characteristics $\boldsymbol{x}_{j}: q_{j}=\boldsymbol{x}_{j} \boldsymbol{\beta}$. The random coefficient $\beta_{i}$ captures consumers' heterogenous tastes for quality. It is assumed to follow a normal distribution with mean $\beta_{0}$ and variance $\sigma^{2}$. Since we cannot separately identify the scale of $\beta_{0}$ and the scale of $\boldsymbol{\beta}$, we normalize the first dimension of $\boldsymbol{\beta}$ to be 1 . The price of product $j$ in period $t$ is denoted by $p_{j t}$.

We include a carrier/year fixed effect in the utility function to capture the average quality and the cost of carrier $c$ 's network service in period $t$ as well as a general time trend in consumers' tastes for smartphones. ${ }^{6}$ We also include a quarter fixed effect to capture seasonality in demand. For simplicity in notation, we denote these two fixed effects by one term $\kappa_{c(j) t}$, where $c(j)$ denotes the carrier for product $j$. The term $\xi_{j t}$ is a demand shock. Finally, the error term $\varepsilon_{i j t}$ captures consumer $i$ 's idiosyncratic taste, which is assumed to be i.i.d. and to follow a type-I extreme value distribution. We normalize the mean utility of the outside option to be 0 . Thus, the utility of the outside option is $u_{i 0 t}=\varepsilon_{i 0 t}$.

Under the type-I extreme value distributional assumption of $\varepsilon_{i j t}$, the market share of the product $j$ is

$$
s_{j}\left(\boldsymbol{q}_{t}, \boldsymbol{p}_{t}, \boldsymbol{\xi}_{t}\right)=\int \frac{\exp \left(\beta_{i} q_{j}-\alpha p_{j t}+\kappa_{c(j) t}+\xi_{j t}\right)}{1+\sum_{j^{\prime} \in \mathcal{J}_{t}} \exp \left(\beta_{i} q_{j^{\prime}}-\alpha p_{j^{\prime} t}+\kappa_{c\left(j^{\prime}\right) t}+\xi_{j^{\prime} t}\right)} d F\left(\beta_{i}\right),
$$

where $\mathcal{J}_{t}$ denotes the set of all products in period $t, \boldsymbol{q}_{t}=\left(q_{j}, j \in \mathcal{J}_{t}\right)$ is a vector of the quality indices of all products in the market, and $\boldsymbol{p}_{t}$ and $\boldsymbol{\xi}_{t}$ are analogously defined.

\subsection{Supply}

On the supply side, we model firms' product decisions and pricing decisions. As mentioned, we take the set of flagship products as given. Thus, in terms of product choice, our model describes

\footnotetext{
${ }^{6}$ We treat carriers' service plans (their features and their prices) as exogenous. We do so for two reasons. First, we do not have data on carriers' service plans. It is also difficult to compare service plans provided by different carriers as they differ in many dimensions. Second, and more importantly, a carrier typically does not redesign its service plans when a new smartphone is introduced to the market. That is, it is plausible to assume that carriers' service plans are exogenous to smartphone firms' product and price decisions.
} 
firms' decisions on their non-flagship products only; but in terms of pricing, our model describes their decisions for all products, flagship or non-flagship. We make this modelling choice because flagship products are typically sold globally. Thus, modelling the product decision on flagship products would require us to have data on the worldwide market. However, studying firms' decisions on flagship products' prices in the U.S. does not impose such a data requirement.

Our model does not explain why a given smartphone is sold through a specific carrier either. We take the carrier choice for each product as exogenous. The carrier choice can be an important decision for flagship products. For example, Sinkinson (2014) studies the exclusive contract between AT\&T and Apple for iPhones. But this decision is presumably less important for non-flagship products. For tractability, we do not model this decision.

Over all, the supply side of the model is described by a static three-stage game. In the first stage, given the set of flagship products in the market, firms choose their non-flagship products. Next, after observing the demand and the marginal cost shocks, firms choose the wholesale prices charged to the carriers. Finally, carriers chooses retail prices, i..e, subsidized prices for smartphones. We describe these three stages backwards.

\subsubsection{Decisions on Prices}

At the third stage, carriers observe the set of products available on each carrier (denoted by $\mathcal{J}_{c t}$ ), the wholesale prices (denoted by $w_{j t}$ ) and the demand shocks $\left(\boldsymbol{\xi}_{t}\right)$. They choose the retail prices $\left(p_{j t}\right)$ to maximize their respective profit. Suppose that the profit that carrier $c$ obtains through its service is $b_{c t}$ per consumer. Carrier $c$ 's profit margin for each unit of product $j$ sold is therefore $p_{j t}+b_{c t}-w_{j t}$. We do not observe $b_{c t}$ or $\omega_{j t}$. But we can back out $\tilde{w}_{j t}=w_{j t}-b_{c(j) t}$ from the first-order condition on the price $p_{j t}$. Specifically, carrier $c$ 's profit maximizing problem is

$$
\max _{p_{j t}, j \in \mathcal{J}_{c t}} \sum_{j \in \mathcal{J}_{c t}} N s_{j}\left(\boldsymbol{q}_{t}, \boldsymbol{p}_{t}, \boldsymbol{\xi}_{t}\right)\left(p_{j t}-\tilde{w}_{j t}\right)
$$

where $N$ is the market size. The first-order condition allows us to back out $\tilde{w}_{j t}$ as:

$$
\tilde{\boldsymbol{w}}_{c t}=\boldsymbol{p}_{c t}+\left(\frac{D \boldsymbol{s}_{c t}}{D \boldsymbol{p}_{c t}}\right)^{-1} \boldsymbol{s}_{c t}
$$

where $\tilde{\boldsymbol{w}}_{c t}=\left(\tilde{w}_{j t}, j \in \mathcal{J}_{c t}\right), \boldsymbol{p}_{c t}=\left(p_{j t}, j \in \mathcal{J}_{c t}\right)$ and $\boldsymbol{s}_{c t}=\left(s_{j}, j \in \mathcal{J}_{c t}\right)$. We denote the equilibrium of this stage by $p_{j t}^{*}\left(\tilde{\boldsymbol{w}}_{t}, \boldsymbol{q}_{t}, \boldsymbol{\xi}_{t}\right)$, where $\tilde{\boldsymbol{w}}_{t}=\left(\tilde{\boldsymbol{w}}_{c t}, c \in \mathcal{C}\right)$.

At the second stage, smartphone firms choose the wholesale prices that they charge carriers after observing the demand shocks and the marginal shocks. We assume that the marginal cost of a product depends on its quality, a time fixed effect, and a product/time-specific shock. ${ }^{7}$ Specifically,

\footnotetext{
${ }^{7}$ Note that a product is a smartphone/carrier combination. Marginal costs may vary across carriers because different radio technologies are used for products sold by different carriers. Moreover, carriers sometimes require
} 
we assume that the marginal cost is $m c_{j t}=\gamma_{t}+\gamma_{1} \exp \left(q_{j}-\bar{q}_{t}\right)+\omega_{j t}$. Let $\tilde{m} c_{j t}=m c_{j t}-b_{c(j) t}$, and $\tilde{\gamma}_{c t}=\gamma_{t}-b_{c t}$. With these notations, we can re-write the marginal cost as

$$
\tilde{m} c_{j t}=\tilde{\gamma}_{c(j) t}+\gamma_{1} \exp \left(q_{j}\right)+\omega_{j t}
$$

The profit that a smartphone firm gets for its product $j$ is therefore

$$
\left(\tilde{w}_{j t}-\tilde{m} c_{j t}\right) N s_{j}\left(\boldsymbol{q}_{t}, \boldsymbol{p}_{t}^{*}\left(\tilde{\boldsymbol{w}}_{t}, \boldsymbol{q}_{t}, \boldsymbol{\xi}_{t}\right), \boldsymbol{\xi}_{t}\right)
$$

Given its products in the market (denoted by $\mathcal{J}_{m t}$ for a smartphone firm $m$ ), a smartphone firm chooses the wholesale prices to maximize its profit. The first-order condition is

$$
s_{j t}+\sum_{j^{\prime} \in \mathcal{J}_{m t}}\left(\tilde{w}_{j^{\prime} t}-\tilde{m} c_{j^{\prime} t}\right)\left(\sum_{j^{\prime \prime} \in \mathcal{J}_{t}} \frac{\partial s_{j^{\prime} t}}{\partial p_{j^{\prime \prime} t}} \frac{\partial p_{j^{\prime \prime} t}^{*}}{\partial \tilde{w}_{j t}}\right)=0
$$

or equivalently,

$$
\tilde{w}_{j t}+\left[\Delta^{-1} \boldsymbol{s}_{m t}\right]_{j t}=\tilde{\gamma}_{c(j) t}+\gamma_{1} \exp \left(q_{j}-\bar{q}_{t}\right)+\omega_{j t}
$$

where $\boldsymbol{s}_{m t}=\left(s_{j}, j \in \mathcal{J}_{m t}\right)$ and $\Delta$ is a $\left|\mathcal{J}_{m t}\right| \times\left|\mathcal{J}_{m t}\right|$ matrix, a typical element of which is $\left(\sum_{j^{\prime \prime} \in \mathcal{J}_{t}} \frac{\partial s_{j^{\prime} t}}{\partial p_{j^{\prime \prime} t}} \frac{\partial p_{j^{\prime \prime} t}^{*}}{\partial \tilde{w}_{j t}}\right)$. Let $\tilde{w}_{j t}^{*}\left(\boldsymbol{q}_{t}, \boldsymbol{\xi}_{t}, \boldsymbol{\omega}_{t}\right)$ be the equilibrium wholesale price minus $b_{c(j) t}$.

\subsubsection{Decisions on Products}

At the first-stage of the model, firms take the set of flagship products in the market as given and choose their set of non-flagship products. There is a fixed cost for every product, ${ }^{8}$ denoted by $F_{j t}$. Nash equilibrium implies that given competitors' product portfolios at the equilibrium, any deviation from a firm's equilibrium product portfolio would generate a lower expected profit for this firm. The expectation is taken over the demand shocks and the marginal cost shocks. Specifically, we consider two types of deviations. First, firm $m$ 's expected profit would be lower if product $j$ in its portfolio (i.e., $j \in \mathcal{J}_{m t}$ ) is removed, i.e.,

$$
E_{\left(\boldsymbol{\xi}_{t}, \boldsymbol{\omega}_{t}\right)} \pi_{m t}\left(\boldsymbol{q}_{t}, \boldsymbol{\xi}_{t}, \boldsymbol{\omega}_{t}\right)-F_{j t} \geq E_{\left(\boldsymbol{\xi}_{t}, \boldsymbol{\omega}_{t}\right)} \pi_{m t}\left(\boldsymbol{q}_{t} \backslash q_{j}, \boldsymbol{\xi}_{t} \backslash \xi_{j t}, \boldsymbol{\omega}_{t} \backslash \omega_{j t}\right) \text { for any } j \in \mathcal{J}_{m t}
$$

where $\pi_{m t}\left(\boldsymbol{q}_{t}, \boldsymbol{\xi}_{t}, \boldsymbol{\omega}_{t}\right)$ be the equilibrium variable profit (at the stage-2 and stage-3 pricing equilibrium) for firm $m$. Second, firm $m$ 's profit would be lower if it adds back product $j$ that it

firms to preload different softwares on a smartphone, which may come with different costs.

${ }^{8}$ Since non-flagship products are behind the technology frontier, we assume that there is no sunk cost of introducing a new non-flagship product. Therefore, similar to Crawford, Shcherbakov and Shum (2015) and Eizenberg (2014), we treat the product choice as static. 
discontinued (i.e., $j \in \mathcal{J}_{m t-1}, j \notin \mathcal{J}_{m t}$ ). The corresponding inequality is

$$
E_{\left(\boldsymbol{\xi}_{t}, \boldsymbol{\omega}_{t}\right)} \pi_{m t}\left(\boldsymbol{q}_{t}, \boldsymbol{\xi}_{t}, \boldsymbol{\omega}_{t}\right) \geq E_{\left(\boldsymbol{\xi}_{t}, \boldsymbol{\omega}_{t}\right)} \pi_{m t}\left(\boldsymbol{q}_{t} \cup q_{j}, \boldsymbol{\xi} \cup \xi_{j t}, \boldsymbol{\omega}_{t} \cup \omega_{j t}\right)-F_{j t} \text { for any } j \in \mathcal{J}_{m t-1}, j \notin \mathcal{J}_{m t} .
$$

\section{Estimation}

\subsection{Instruments and Estimation Procedure}

We estimate the parameters on the demand side and the parameters in the smartphone marginal cost function using the Generalized Method of Moments. The demand estimation follows Berry, Levinsohn and Pakes (1995). Following the literature, our instrumental variables are based on characteristics of products of the same carrier and the same smartphone firm, of the same carrier but different firms, and of different carriers. Based on the demand estimation, we construct the quality index of a product as a linear combination of the product's characteristics. The estimation of the marginal cost parameters is based on the first-order condition with respect to the wholesale price (equation (8)).

As for the fixed cost, we use inequalities (9) and (10) to obtain either an upper bound or a lower bound for product-month combinations in the data. Specifically, we obtain an upper bound for each product in the market, i.e., any $j \in \mathcal{J}_{t}$, using inequality (9), and a lower bound of the fixed cost $F_{j t}$ for each dropped product, i.e., any $j \in \mathcal{J}_{t-1}, j \notin \mathcal{J}_{t}$ using inequality (10).

\subsection{Estimation Results}

Table 5 reports the estimation results on demand and marginal cost. The estimation results indicate that consumers on average favor products with a longer battery talk time, a higher camera resolution, a higher-generation chipset, and a larger screen. For example, an increase in the battery talk time by one hour is equivalent to a decrease in the price by 7 dollars for an average consumer. Similarly, an increase in the camera resolution by 1 megapixel is equivalent to a decrease in the price by 9 dollars, and an increase in the screen size by 0.1 inch is equivalent to a decrease in the price by 13 dollars. The estimation results also suggest that, ceteris paribus, consumers on average prefer a lighter smartphone, though the estimate of this coefficient is statistically insignificant. The estimated standard deviation of consumers' taste for quality is about $1 / 3$ of the average taste, suggesting that consumers are heterogenous in their willingness-to-pay for quality. We include three brand dummies (Apple, BlackBerry and Samsung) and group all other brands as the baseline brand in the utility function. According to our estimates, there is a large Apple premium (more than 400 dollars), followed by BlackBerry and then Samsung. ${ }^{9}$ Our estimation results also suggest

\footnotetext{
${ }^{9}$ Note that even though the estimated BlackBerry-dummy coefficient is larger than that of Samsung, considering the product characteristics, the average quality of Samsung products in month is generally higher than that of
} 
that there is an advantage of being a flagship product.

Table 5: Estimation Results

\begin{tabular}{|c|c|c|}
\hline & Parameter & Std. Error \\
\hline \multicolumn{3}{|l|}{ Demand } \\
\hline \multicolumn{3}{|l|}{ Quality coefficient } \\
\hline Battery talk time (hours) & $0.050^{* * *}$ & 0.013 \\
\hline Camera resolution (megapixel) & $0.066^{* * *}$ & 0.032 \\
\hline Chipset generation 2 & $0.468^{* * *}$ & 0.124 \\
\hline Chipset generation 3 & $0.760^{* * *}$ & 0.162 \\
\hline Chipset generation 4 & $1.240^{* * *}$ & 0.224 \\
\hline Chipset generation 5 & $1.929^{* * *}$ & 0.316 \\
\hline Screen size (inch) & 1 & \\
\hline Weight (gram) & -0.0019 & 0.0015 \\
\hline \multicolumn{3}{|l|}{ Quality random coefficient } \\
\hline Mean & $0.742^{* * *}$ & 0.126 \\
\hline Std. Dev. & $0.255^{* * *}$ & 0.081 \\
\hline Price & $-0.006^{* * *}$ & 0.001 \\
\hline Apple & $2.653^{* * *}$ & 0.089 \\
\hline BlackBerry & $1.145^{* * *}$ & 0.112 \\
\hline Samsung & $0.279^{* * *}$ & 0.065 \\
\hline Flagship? & $0.730^{* * *}$ & 0.065 \\
\hline Carrier/year and quarter dummies & \multicolumn{2}{|c|}{ Yes } \\
\hline \multicolumn{3}{|c|}{ Marginal Cost $(\$)$} \\
\hline Exp(quality/10) & $136.731^{* * *}$ & 0.127 \\
\hline Apple & $-23.050^{* * *}$ & 0.092 \\
\hline BlackBerry & $32.152^{* * *}$ & 0.063 \\
\hline Samsung & $-4.545^{* * *}$ & 0.051 \\
\hline Carrier/year dummies & \multicolumn{2}{|c|}{ Yes } \\
\hline
\end{tabular}

Table 6 reports the price elasticities and semi-elasticities for five top-sales products on AT\&T in March 2013. These five products are Motorola's Atrix HD, Samsung's Galaxy S III and Apple's iPhone 4, iPhone 4s and iPhone 5. In Table 6 , the entry in Row $i$ and Column $j$ gives the percentage change in market share of product $j$ with a one-percentage change (elasticity) or a one-dollar change (semi-elasticity) in the price of product $i$. From this table, we can see that, for these five products, a one-dollar increase in a product's price leads to an around $0.5 \%$ decrease in the respective product's demand. Unsurprisingly, the own price elasticities and semi-elasticities are larger than the cross elasticities and semi-elasticities.

We construct the quality index for each product based on the estimated coefficients of the product characteristics. Table 7 reports the elasticities of quality, again for the five products in Table 6, using the estimated quality index. Across all five models, we see that a one percent increase in the quality index corresponds to a $5 \%$ to $7 \%$ of sales increase.

BlackBerry products especially later in the sample. 
Table 6: Demand Elasticities with Respect to Price

\begin{tabular}{lccccc}
\hline \multicolumn{5}{c}{ Price Elasticities } \\
\hline & Atrix HD & Galaxy S III & iPhone 4 & iPhone 4s & iPhone 5 \\
Atrix HD & -0.174 & 0.013 & 0.001 & 0.017 & 0.066 \\
Galaxy S III & 0.002 & -1.059 & 0.001 & 0.017 & 0.068 \\
iPhone 4 & 0.001 & 0.010 & -0.033 & 0.014 & 0.051 \\
iPhone 4s & 0.001 & 0.011 & 0.001 & -0.575 & 0.056 \\
iPhone 5 & 0.001 & 0.012 & 0.001 & 0.016 & -1.161 \\
\hline \multicolumn{7}{c}{ Price Semi-elasticities } \\
\hline Atrix HD & Atrix HD & Galaxy S III & iPhone 4 & iPhone 4s & iPhone 5 \\
Galaxy S III & -0.559 & 0.007 & 0.012 & 0.016 & 0.030 \\
iPhone 4 & 0.005 & -0.557 & 0.012 & 0.017 & 0.031 \\
iPhone 4s & 0.004 & 0.005 & -0.553 & 0.013 & 0.024 \\
iPhone 5 & 0.004 & 0.006 & 0.011 & -0.549 & 0.026 \\
\hline
\end{tabular}

Note: March 2013 AT\&T.

Table 7: Elasticity of Quality

\begin{tabular}{lccccc}
\hline & Atrix HD & Galaxy S III & iPhone 4 & iPhone 4s & iPhone 5 \\
\hline Atrix HD & 7.093 & -0.089 & -0.110 & -0.165 & -0.359 \\
Galaxy S III & -0.062 & 7.316 & -0.112 & -0.169 & -0.37 \\
iPhone 4 & -0.045 & -0.066 & 4.984 & -0.136 & -0.278 \\
iPhone 4s & -0.050 & -0.073 & -0.100 & 5.605 & -0.304 \\
iPhone 5 & -0.057 & -0.083 & -0.106 & -0.158 & 6.310 \\
\hline
\end{tabular}

Note: March 2013 AT\&T.

To see the evolution of smartphone quality over time, we multiply the quality index by the mean taste for quality, and then add the brand fixed effect. We plot the maximum of this index across all products in a month over time in Figure 1 to see the change in the quality frontier over time. We also plot the median quality in the same figure. Finally, we plot the maximum of this index for Apple and Samsung, i.e., their quality frontiers. The Apple highest-quality line perfectly coincides with the industry highest-quality line. The frontier experiences a discrete jump whenever a new iPhone product is introduced. This means that our estimates confirm the belief that the quality frontier is driven by the iPhone products. Also consistent with the common belief, we find that the median quality has risen over time. In fact, the median quality stays at a relatively constant distance behind the frontier. As for the quality frontiers of Apple and Samsung, Figure 1 shows that Samsung has narrowed the quality gap between its smartphone products and iPhones.

On the supply side, we find that marginal cost is increasing in product quality. Though not reported in Table 5, the estimated carrier/year fixed effects indicate that marginal cost is decreasing over time. But note that the marginal cost in the estimation equation (8) is in fact a smartphone firm's marginal cost subtracted by a carrier's service profit. The estimated time trend therefore 
Figure 1: Smartphone Quality over Time

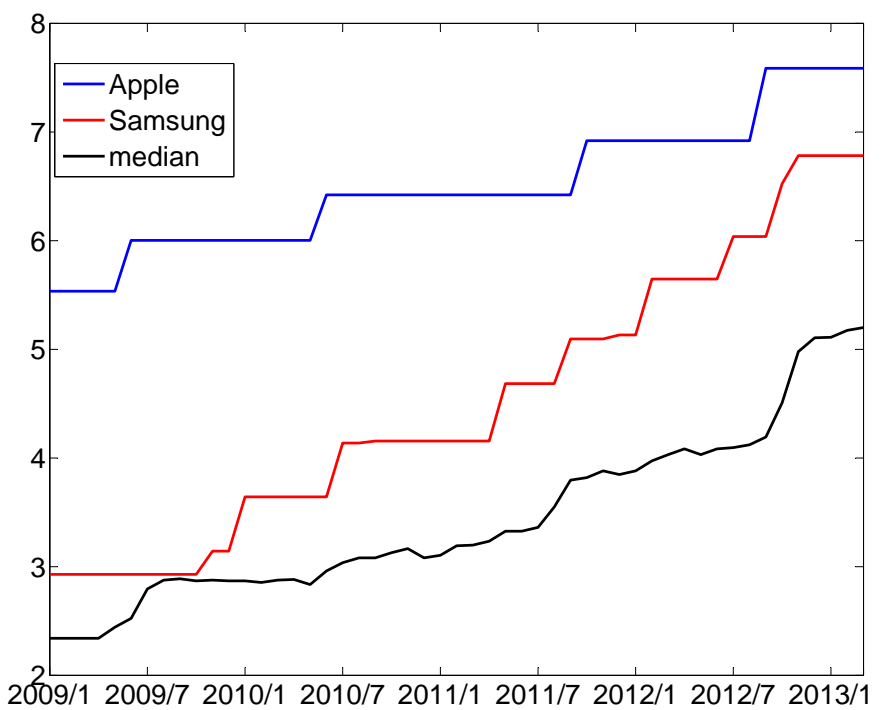

lumps the time trend in marginal cost for a smartphone firm with the time trend in the service profit for a carrier. Similarly, if the average profit that a carrier obtains through its service plans varies by the smartphone brand, the brand dummy coefficients capture not only the brand fixed effect in the marginal cost but also this variation in a carrier's service profit.

Based on the estimates of the demand and marginal cost functions, we obtain an upper bound of the fixed cost for all product-month combinations such that the given product is in the given month, i.e., we obtain an upper bound of $F_{j t}$ for all $j \in \mathcal{J}_{t}$. We also obtain a lower bound of $F_{j t}$ for dropped products, i.e., all $j \in \mathcal{J}_{t-1}, j \notin \mathcal{J}_{t}$. The average lower bound, averaged across all $j t$ such that $j \in \mathcal{J}_{t-1}, j \notin \mathcal{J}_{t}$, is 5.90 million dollars. The average upper bound, averaged across all $j \in \mathcal{J}_{t}$, is 7.14 million dollars. Figure 2 plots these bounds (lower bounds in the left panel and upper bounds in the right panel) against the quality of the corresponding product. This figure suggests that the fixed cost is positively correlated with the quality of a product.

\section{Counterfactual Simulations}

\subsection{Are there too few or too many products?}

There are two reasons why product offerings in an oligopoly market are inefficient. First, given the competitors' products, a firm will offer a product as long as the marginal profit from doing so is positive. But part of this marginal profit may come from business stealing. Since the firm does not take into account this potentially negative externality when making its products decision, there might be too many products from a welfare point of view. Second, consumer surplus is not part 
(a) Lower Bound

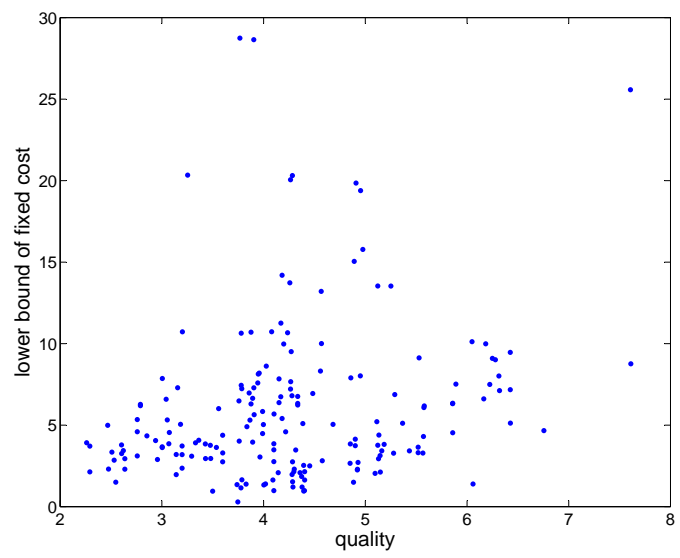

(b) Upper Bound

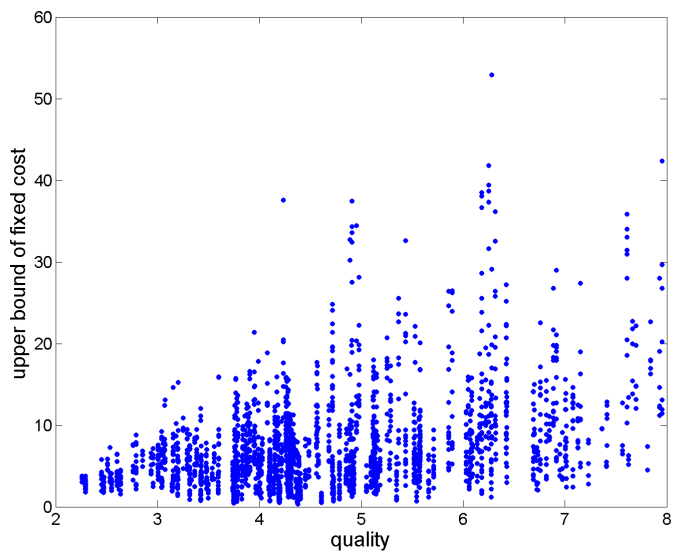

of a firm's objective function. Given that consumer surplus is presumably increasing in product variety, tere might also be too few products. Therefore, this is an empirical question.

To address this question, we first conduct the following counterfactual simulation. For each month between January and March 2013, the last three months of our data, we drop the lowestquality product in the month, solve for the equilibrium wholesale prices and retail prices, and then compute the corresponding consumer surplus, smartphone firms' profits net of fixed costs, and carriers' profits. In Table 8, we report the changes in these welfare measures, and compare them with the saving in fixed costs due to less products. Specifically, in the first three rows, we report changes in consumer surplus, carrier surplus (the sum of carriers' profits) and smartphone producer surplus net of fixed costs (the sum of smartphone firms' profits net of fixed costs). In the last row of Table 8, we report the upper bound of the change in fixed costs, which is the upper bound of the lowest-quality product's fixed cost.

Table 8: Welfare Changes when the Lowest-quality Product is Dropped

\begin{tabular}{lrrr}
\hline & Jan 2013 & $\begin{array}{c}\text { Feb 2013 } \\
\text { (million } \$)\end{array}$ & Mar 2013 \\
\hline \hline$\Delta$ (consumer surplus) & -0.37 & -0.36 & -0.34 \\
$\Delta$ (carrier surplus) & -0.27 & -0.27 & -0.24 \\
$\Delta$ (smartphone producer surplus net of fixed costs) & -0.23 & -0.22 & -0.22 \\
upper bound of savings in fixed costs & 0.36 & 0.35 & 0.33 \\
\hline
\end{tabular}

From Table 8, we can see that consumers are indeed worse off due to the decreased product offerings. Carriers' profits also drop. The comparison between the third row and the last row shows that if the fixed cost is at its upper bound, the total smartphone producer surplus increases after the 
lowest-quality product is dropped, confirming the intuition that by not internalizing the business stealing effect, firms in a competitive market may offer excessive product variety especially if the fixed cost is high. However, this effect is dominated by the effect of product variety on consumer surplus. Dropping the lowest-quality product in a month reduces consumer surplus. Summing over the four rows of Table 8, we can see that even considering the maximum possible saving in the fixed cost, dropping the lowest-quality product leads to a decrease in the total welfare.

Table 9 presents the results when we drop the lowest-quality product of each smartphone firm. The changes in all welfare measures are larger than the case of dropping only one product. The main conclusion remains the same: the decrease in consumer surplus dominates the increase in smartphone producer surplus even considering the maximum possible saving in the fixed cost from dropping products. In summary, these counterfactual simulation results suggest that dropping products do not improve total welfare, i.e., there are not too many products. But are there too few? In what follows, we repeat the exercise by adding a product, specifically, the highest-quality non-flagship product that was dropped from last month to this month.

Table 9: Welfare Changes when the Lowest-quality Product of Each Smartphone Firm is Dropped

\begin{tabular}{lrrr}
\hline & Jan 2013 & $\begin{array}{c}\text { Feb 2013 } \\
\text { (million \$) }\end{array}$ & Mar 2013 \\
\hline \hline$\Delta$ (consumer surplus) & -15.97 & -13.17 & -15.10 \\
$\Delta$ (carrier surplus) & -12.41 & -10.99 & -12.14 \\
$\Delta$ (smartphone producer surplus net of fixed costs) & -8.74 & -6.56 & -7.63 \\
upper bound of savings in fixed costs & 15.86 & 13.28 & 15.14 \\
\hline
\end{tabular}

Table 10 reports the simulation result. Not surprisingly, consumers are better-off with the additional product in the market, carriers also earn more profits, and smartphone producer surplus net of fixed costs increases. For the added product, which is a discontinued product, we can only obtain a lower bound on its fixed cost. This is because the fact that it is discontinued by a firm implies a lower bound on its fixed cost, but does not provide information on its upper bound. Therefore, we can only obtain an upper bound on the total welfare gain from adding these products. By summing up the first three row of Table 10 and comparing the sum with the lower bound of the fixed cost, we can see that as long as the fixed cost is not too high (i.e., not more than 4 to 5 times of its lower bound), adding these products is welfare enhancing.

Overall, our simulation results from dropping products and adding products suggest that there are two few products. As mentioned, because firms do not internalize the potentially negative externality of business stealing, there might be too many products. But on the other hand, firms do not consider consumer surplus either, which may lead to inefficiently low number of products. In the end, our results suggest that the second effect dominates the first effect. 
Table 10: Welfare Changes when the Highest-quality Dropped Product is Added Back

\begin{tabular}{lrrr}
\hline & Jan 2013 & $\begin{array}{c}\text { Feb 2013 } \\
\text { (million \$) }\end{array}$ & Mar 2013 \\
\hline \hline$\Delta$ (consumer surplus) & 7.56 & 3.00 & 3.74 \\
$\Delta$ (carrier surplus) & 6.13 & 2.42 & 2.76 \\
$\Delta$ (smartphone producer surplus net of fixed costs) & 1.93 & 1.25 & 1.60 \\
lower bound of added fixed costs & 4.30 & 1.39 & 1.49 \\
\hline
\end{tabular}

\subsection{How does competition affect product offerings?}

To study how competition affects product offerings, we simulate the effect of a hypothetical merger between Samsung and LG in March 2013. Samsung and LG are the second and the third largest smartphone firms in terms of sales in this month. In this exercise, we compute the postmerger product-choice equilibrium and pricing equilibrium. In theory, the product-choice action space for a firm is very large. A firm can choose to drop any set of products or adding any set of products after the merger. To keep the problem tractable, in the counterfactual simulation, we restrict the set of potential products for each firm to be this firm's products in the data in either this month or last month. In other words, after the merger, a firm can drop any products or add back any products that it has discontinued.

Even with this restricted set of potential products, the choice set for a firm can be very large. This is because a smartphone firm chooses a product portfolio, which is a subsect (of any size) of the potential products. For example, Samsung has 16 non-flagship products and LG has 11 in March 2013. The choice set for this merged firm consists of any 1 product out of the 27 products, any 2 products out of these 27 products, any 3 products, so on and so forth. This choice set has more than 134 million such product portfolios. To deal with this problem, we use a heuristic approach similar to one suggested by Belloni, Freund, Selove and Simester (2008) to compute a firm's optimal product portfolio given competitors' product portfolios. This approach is then embedded in the best-response iteration to solve for the post-merge equilibrium. To describe our algorithm more in detail, let $\mathcal{J}_{m}^{0}$ be firm $m$ 's "product portfolio" before the merger. For each $m=1, \ldots, M$, we compute its best product portfolio $\mathcal{J}_{m}^{1}$ when other firms' product portfolios are given by $\mathcal{J}_{m^{\prime}}^{0}$ for $m^{\prime} \neq m$. In the next round of the best-response iteration, we find each firm's best product portfolio $\mathcal{J}_{m}^{2}$ when other firms' product portfolios are given by $\mathcal{J}_{m^{\prime}}^{1}$ for $m^{\prime} \neq m$. We repeat this process until it converges. To find the best response for $m$ in the $k^{t h}$ round of the best-response iteration, we start with $\mathcal{J}_{m}^{k}$ and compute its profit from each one of the following deviations from $\mathcal{J}_{m}^{k}$ : a product in $\mathcal{J}_{m}^{k}$ is removed or a potential product not in $\mathcal{J}_{m}^{k}$ is added. There are $N_{m}$ such deviations, where $N_{m}$ is the number of potential products for $m$. Each deviation differs from $\mathcal{J}_{m}^{k}$ in only one product. Let $\mathcal{J}_{m}^{k(1)}$ be highest-profit deviating product portfolio. If firm $m$ 's profit corresponding to $\mathcal{J}_{m}^{k(1)}$ is smaller than that corresponding to $\mathcal{J}_{m}^{k}$, this procedure stops and returns $\mathcal{J}_{m}^{k}$ as the best 
response. Otherwise, we compute $m$ 's profit from any one-product deviation from $\mathcal{J}_{m}^{k(1)}$ by adding a potential product to or dropping a product from $\mathcal{J}_{m}^{k(1)}$. We continue this process until firm $m$ 's profit does not increase any more. This is how we find each firm's best response in each round of the best-response iteration.

Table 11 reports changes in the number of products, consumer surplus, carrier surplus and smartphone producer surplus. Since we obtain only a bound on the fixed cost, we report the results using different values of fixed costs within the bounds. Specifically, for each product in the data (i.e., $j \in \mathcal{J}$ ), we have obtained an upper bound of its fixed cost (denoted by $\bar{F}_{j t}$ ). For such a product, we set the lower bound of its fixed cost to be the average lower bound (denoted by $\underline{F}$ ). This average is taken over all lower bounds we obtain in Section 4 . However, to ensure that the range is not too wide, we replace this average by half of the upper bound if the former is smaller than the latter. In other words, the lower bound is $\max \left(\underline{F}, 0.5 \bar{F}_{j t}\right)$. Similarly, for each potential product not in the data, we have obtained a lower bound of its fixed cost. We set the upper bound to be the average upper bound, which is again taken over all upper bounds we have obtained, but replace it by 1.5 of the lower bound if the range is too wide. In other words, the range is $\left[\underline{F}_{j t}, \min \left(\bar{F}, 1.5 \underline{F}_{j t}\right)\right]$, where $\bar{F}$ is the average upper bound. In Table 11 , we randomly draw 5 fixed cost values within the range of each potential product's fixed cost, and report the simulation results corresponding to all five sets of draws.

Table 11: The Effect of Samsung-LG Merger in March 2013

\begin{tabular}{lrrrrr}
\hline Fixed cost draw & Draw 1 & Draw 2 & Draw 3 & Draw 4 & Draw 5 \\
\hline \hline$\Delta$ (\# of products) & 5 & 2 & 2 & 3 & 3 \\
$\Delta$ (sales-weighted avg price) & 9.28 & 8.02 & 5.60 & 8.92 & 6.00 \\
$\Delta$ (total sales) & $-204,028$ & $-80,151$ & $-111,262$ & $-101,747$ & $-109,391$ \\
$\Delta$ (consumer surplus in million $\$)$ & -64.21 & -40.47 & -46.45 & -44.31 & -45.84 \\
$\Delta$ (carrier surplus in million\$) & -36.18 & -24.90 & -29.27 & -27.15 & -29.51 \\
$\Delta$ (smartphone producer surplus in million\$) & 24.29 & 16.56 & 21.48 & 18.37 & 19.47 \\
\hline
\end{tabular}

Table 11 shows that after the merger, the number of products decreases under all five random draws of the fixed costs. Specifically, in all five simulations, the merged firm drops products while the other firms do not adjust their product portfolios. ${ }^{10}$ Also, not surprisingly, prices increase. The sales-weighted average retail price increases by around 8 dollars. This is largely due to increases in the prices of the merged firm's products. Though not reported in the Table, we find that the sales-weighted average retail price of these products increases by more than 15 dollars for all five simulations. The decreased product offerings and the increased retail prices lead to a reduction in consumer surplus by around 40 to 60 million dollars depending on the fixed cost draws. Carriers are also worse off. This is because the decrease in the product offerings shifts demand to the left.

\footnotetext{
${ }^{10}$ This result of other firms not adjusting their product portfolios might be due to the restriction we impose on their potential products. In another simulation, we plan to expand the set of potential products.
} 
Moreover, the sales-weighted wholesale prices increase. The total smartphone producer surplus increases. But the overall welfare decreases by nearly 50 to more than 70 million dollars.

In summary, results from this counterfactual simulation show that a reduction in competition leads to a decrease in product offering in the market, which leads to a decline in consumers surplus and carrier surplus, and a reduction in the overall welfare despite an increase in smartphone producer surplus. Combining with our results in Section 5.1 that there are too few products in the market, these results suggest that merger policies should be stricter when we take into account the effect of merger on product variety.

\section{Conclusion}

In this paper, we study the welfare implication of product proliferation and the effect of competition on product proliferation in the U.S. smartphone market. To this end, we develop and estimate a model for the demand and supply of smartphones. We conduct counterfactual simulations where we add or drop products to answer the question of whether there are too few or too many products in the market, and a merger simulation to study the effect of competition on product offerings. We find that there are too few products in the market and a reduction in competition decreases product offering and leads to a welfare decline. These results suggest that we should be more concerned with mergers when we take into account their effect on product choices.

We conclude the paper with highlighting a few caveats of the paper. First, similar to many papers in the endogenous product choice literature, this paper sets up a static model to describe consumer demand and firm behavior. On the supply side, this modelling choice is somewhat justifiable because we focus on firms' decisions of non-flagship products, which presumably do not involve a large sunk cost such as the R\&D cost. However, due to frictions such as switching costs, consumers may be "dynamic", which will lead to firm dynamic behavior. For example, it might be costly for consumers to switch from one carrier to another. Note that in a reduced-form way, our carrier/year fixed effect in the utility function captures the average switching cost for consumers buying from a carrier. For instance, the fixed effect for Verizon in a year captures its opponents' market shares in the previous year, which determines the proportion of consumers who have to pay switching cost to buy a Verizon product this year. Therefore, this fixed effects somewhat captures the average switching cost for consumers to buy a Verizon product. That said, we do keep the fixed effects constant in the counterfactual simulations and therefore cannot discuss industry dynamics.

Second, as mentioned, our model does not explain why a product is sold through a specific carrier. This means that we cannot discuss the effect of competition on the carrier choice for each product, which may affect the pricing equilibrium, and thus also affect smartphone firms' product offerings. The carrier choice is particularly important when switching costs are prominent. Unfortunately, without individual-level data which may help us to reliably identify the switching 
cost and without information on the contracts between a carrier and a smartphone firm, it is difficult for us to discuss the carrier choice. We therefore leave this for future research.

\section{References}

Belloni, Alexandre, Robert Freund, Matthew Selove, and Duncan Simester (2008), "Optimizing product line designs: Efficient methods and comparisons." Management Science, 54, 1544-1552.

Berry, Steven, Alon Eizenberg, and Joel Waldfogel (forthcoming), "Horizontal and verticalproduct variety in radio markets." RAND Journal of Economics.

Berry, Steven, James Levinsohn, and Ariel Pakes (1995), "Automobile prices in market equilibrium." Econometrica, 63, 841-90.

Chu, Chenghuan Sean (2010), "The effect of satellite entry on cable television prices and product quality." RAND Journal of Economics, 41, 730-764.

Crawford, Gregory (2012), "Accommodating endogenous product choices: A progress report." International Journal of Industrial Organization, 30, 315-320.

Crawford, Gregory S., Oleksandr Shcherbakov, and Matthew Shum (2015), "The welfare effects of endogenous quality choice in cable television markets." Cepr discussion paper.

Crawford, Gregory S. and Ali Yurukoglu (2012), "The Welfare Effects of Bundling in Multichannel Television Markets." American Economic Review, 102, 643-85.

Draganska, Michaela, Michael Mazzeo, and Katja Seim (2009), "Beyond plain vanilla: Modeling joint product assortment and pricing decisions." Quantitative Marketing and Economics, 7, 105146.

Eizenberg, Alon (2014), "Upstream innovation and product variety in the U.S. home PC market." Review of Economic Studies, 81, 1003-1045.

Fan, Ying (2013), "Ownership Consolidation and Product Characteristics: A Study of the US Daily Newspaper Market." American Economic Review, 103, 1598-1628.

Johnson, Justin P. and David P. Myatt (2003), "Multiproduct Quality Competition: Fighting Brands and Product Line Pruning." American Economic Review, 93, 748-774.

Mazzeo, Michael (2002), "Product choice and oligopoly market structure." The RAND Journal of Economics, 33, 221-242.

Nosko, Chris, "Competition and quality choice in the cpu market." working paper, University of Chicago. 
Seim, Katja (2006), "An empirical model of firm entry with endogenous product-type choices." RAND Journal of Economics, 37, 619-640.

Shen, Jian, Huanxing Yang, and Lixin Ye (forthcoming), "Competitive nonlinear pricing and contract variety." Journal of Industrial Economics.

Sinkinson, Michael (2014), "Pricing and entry incentives with exclusive contracts: Evidence from smartphones." working paper, University of Pennsylvania.

Sweeting, Andrew (2013), "Dynamic product positioning in differentiated product markets: The effect of fees for musical performance rights on the commercial radio industry." Econometrica, 81, 1763-1803.

Watson, Randal (2009), "Product variety and competition in the retail market for eyeglasses." Journal of Industrial Economics, 57, 217-251. 